\title{
A Stochastic Model of the Electrically Stimulated Auditory Nerve: Pulse-Train Response
}

\author{
Ian C. Bruce,* Member, IEEE, Laurence S. Irlicht, Member, IEEE, Mark W. White, Member, IEEE, \\ Stephen J. O’Leary, Scott Dynes, Eric Javel, and Graeme M. Clark
}

\begin{abstract}
The single-pulse model of the companion paper [1] is extended to describe responses to pulse trains by introducing a phenomenological refractory mechanism. Comparisons with physiological data from cat auditory nerve fibers are made for pulse rates between 100 and 800 pulses/s. First, it is shown that both the shape and slope of mean discharge rate curves are better predicted by the stochastic model than by the deterministic model. Second, while interpulse effects such as refractory effects do indeed increase the dynamic range at higher pulse rates, both the physiological data and the model indicate that much of the dynamic range for pulse-train stimuli is due to stochastic activity. Third, it is shown that the stochastic model is able to predict the general magnitude and behavior of variance in discharge rate as a function of pulse rate, while the deterministic model predicts no variance at all.
\end{abstract}

Index Terms - Auditory nerve, cochlear implant, functional electrical stimulation, pulse-train response, population response, refractory effect, renewal process, sensory prosthesis, stochastic model.

\section{INTRODUCTION}

I $\mathrm{N}$ [1], we presented a stochastic model of auditory nerve (AN) response to single electrical pulses, following the conceptual approach used in [2]-[4]. In [5]-[8], we have gone on to show that the stochastic single-pulse model better predicts a range of psychophysical measures of loudness than the equivalent deterministic model does. In this paper, we extend the model to describe responses to pulse-train stimuli. The final model, although more complex than the single-pulse

Manuscript received October 20, 1997; revised January 5, 1999. This work was supported by the Human Communication Research Centre, The University of Melbourne, the Bionic Ear Institute, and the CRC for Cochlear Implants, Speech and Hearing Research. Asterisk indicates corresponding author.

*I. C. Bruce was with the Bionic Ear Institute and the Department of Otolaryngology, The University of Melbourne, East Melbourne VIC 3002, Australia. He is now with the Department of Biomedical Engineering, 505 Traylor Bldg., Johns Hopkins University, 720 Rutland Ave, Baltimore, MD 21205 USA (e-mail: ibruce@bme.jhu.edu).

L. S. Irlicht was with the Bionic Ear Institute and the Department of Otolaryngology, The University of Melbourne, East Melbourne, VIC 3002, Australia. He is now with County Investment Management, Melbourne VIC 3000 , Australia.

M. W. White is with Department of Electrical and Computer Engineering, North Carolina State University, Raleigh, NC 27695 USA.

S. J. O'Leary and G. M. Clark are with the Bionic Ear Institute and the Department of Otolaryngology, The University of Melbourne, East Melbourne VIC 3002, Australia.

S. Dynes is with the Eaton-Peabody Laboratory, Massachusetts Eye and Ear Infirmary, Boston, MA 02114 USA.

E. Javel is with the Department of Otolaryngology, University of Minnesota, Minneapolis, MN 55455 USA.

Publisher Item Identifier S 0018-9294(99)03978-6. model, is still computationally efficient and can be fitted easily to the statistics of AN parameters collected from physiological studies.

Furthermore, this simple model is particularly valuable because it is composed of several "conceptual components" (e.g., a stochastic component, a refractory component). The impact of various modifications or exclusions of the components can be easily explored with such models-we will illustrate the utility of this feature.

In Section II, we describe our analysis of the physiological data. In Section III, we present the pulse-train model and investigate response properties of the model at a range of pulse rates. In particular, we compare the behavior of the model with and without the stochastic component. In Section IV, we describe the effects of pulse-train stimulation on the model of total AN response developed in [1]. Finally, in Section V, we discuss further physiological and modeling studies that would help in refining and extending our model.

\section{METHODS}

The physiological data presented in this paper are singlefiber cat AN data from [9] (see [1] for a summary of the physiological methods). Along with Javel et al.'s analysis of mean discharge rate in response to trains of evenly timed, uniform, biphasic current pulses, we present a previously unpublished analysis of this data set. The variance in discharge rate is estimated by calculating the variance in the number of discharges recorded in response to repeated presentation of a 100-ms pulse train. This analysis is examined further in [10].

\section{Pulse-Train Response}

Although the investigation of single-pulse responses is useful in the development and verification of a neural model [1], single-pulse stimuli are not the usual output from cochlear implants. Most cochlear implant speech processing strategies typically utilize trains of pulses at rates higher than 100 pulses/s (pps) and train durations up to hundreds of milliseconds. Therefore, a practical model must accurately describe the neural response to trains of current pulses at a range of pulse rates and train durations.

\section{A. Stochastic Model of Pulse-Train Response}

In the case of pulse trains, the time-dependent nature of neural response to current pulses means that the response 


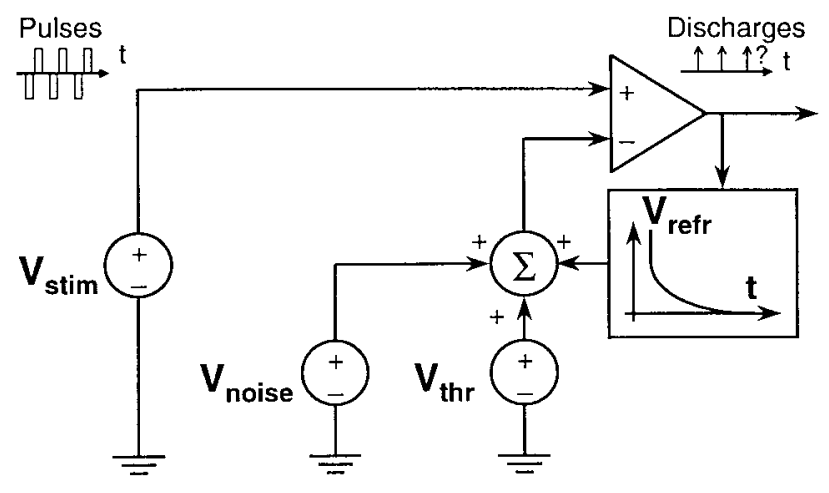

Fig. 1. Stochastic model of pulse-train response. See text for explanation of circuit components.

to any one pulse in the train will be dependent on how the neuron has responded to the previous pulses in the pulse train. Such dependencies are commonly referred to as interpulse interactions.

The major form of interpulse interaction seen in physiological data is known as the refractory effect, whereby after an action potential is generated there is some time over which the neuron is either unable or less able to be driven to discharge again [3], [11]-[13]. The time over which the neuron is subject to refractory effects is notionally divided into the absolute refractory period and the relative refractory period. The former occurs immediately after action potential generation, and during this time it is not possible to produce another discharge. Following the absolute refractory period is the relative refractory period, during which the neuron is harder to drive to discharge than when it is outside the refractory period.

We are able to approximate this behavior by introducing a phenomenological mechanism to our model that describes the end result of the refractory effects on the discharge probability. Following our approach in [1], the single-fiber model presented in this paper is defined in the form of an electrical circuit, suitable for Monte Carlo simulations. To the single-pulse model of [1] we add a refractory potential $V_{\text {refr }}$ as shown in Fig. 1. Following an action potential, the 'threshold' with which the stimulus potential is compared is raised over the refractory period by some chosen function.

The refractory function $V_{\text {refr }}$ will have a value of infinity for the absolute refractory period, and will then generally fall smoothly to zero over the relative refractory period. We, therefore, make two assumptions regarding the behavior of the refractory function.

1) $V_{\text {refr }}$ is assumed to be monotonically decreasing, i.e., it falls from its maximum value at the beginning of the refractory period to its minimum value at the end of the refractory period without any transitory increases.

2) The refractory effects are assumed to be of finite duration, i.e., $V_{\text {refr }}$ returns to a value of zero within a finite time.

Refractory effects may also change the behavior of the membrane noise. It appears that the variance of $V_{\text {noise }}$ may increase with small, sustained depolarizations [1], [2]. The behavior of the membrane noise during the larger depolarization that occurs as a result of an action potential is not well understood. This requires a more thorough investigation of the behavior of ionic channels during and after action potential generation. Some physiological data and modeling results indicate that the noise only increases significantly during depolarizations that do not generate an action potential [13, Fig. 5-7], whereas others suggest that the membrane noise could increase greatly during a segment of the refractory period under some conditions [14], [15]. Due to the uncertainty about the exact nature of these phenomena, we will not attempt incorporate them into the model at this stage. Consequently, the standard deviation of $V_{\text {noise }}$ remains constant during the refractory period, i.e., $\sigma$ of $V_{\text {noise }}=\mathrm{RS} \times V_{\mathrm{thr}} \cdot{ }^{1}$

An excitatory interpulse effect has also been observed if the depolarization caused by a pulse is not great enough to produce an action potential. In this case, any pulse following this subthreshold "conditioner" pulse will have a reduced threshold [13], [16]. These excitatory effects generally last for approximately half a millisecond, but may have longer time constants in small diameter fibers [17, Ch. IV]. This effect requires further investigation, and could be added to our model when sufficient information becomes available.

Autocorrelation of the membrane noise could also produce interpulse interactions, i.e., the noise potential during one pulse may be correlated with the noise potential during the preceding and following pulses in the pulse train, such that their responses are not independent. Such effects could be investigated electrophysiologically or by using a Hodgkin-Huxley-type model including stochastic activity [18]-[20], but such an investigation would be extremely difficult either experimentally or computationally and is beyond the scope of this paper. Therefore, we use a simplifying approximation that $V_{\text {noise }}$ is considered to be uncorrelated between pulses.

Finally, for the purpose of numerical evaluation the model is discretized, i.e., each pulse $n$ is divided into a number of discrete bins of equal time. The level of discretization, i.e., number of bins per pulse, will have an effect at higher pulse rates where a number of pulses fall within the relative refractory period. The number of bins required is dependent on the slope of the refractory function. We used ten bins per pulse as our discretization level in computing our simulation results, because we found that increasing the number of bins beyond ten had negligible effect.

\section{B. Analytical Approximation of Stochastic Model}

The stochastic model presented in Section III can be described as a renewal process [21], [22]. In a renewal process, the waiting times between successive occurrences of an event are mutually independent random variables having the same distribution [22, p. 303]. Our model qualifies as a renewal process, because after each discharge it begins anew without any "memory" of prior discharges.

\footnotetext{
${ }^{1}$ Relative spread (RS) is defined as the standard deviation of the noise $(\sigma)$ divided by the threshold for the single-pulse response. Refer to [1] for further explanation of this nomenclature.
} 
Using renewal process theory, exact analytical expressions for the mean and variance in discharge rate can be derived for our stochastic model in the case of a pulse train of infinite duration. We approximate the actual finite pulse train of evenly timed, uniform pulses by a pulse train of infinite duration. The full mathematical derivation of our analytical approximation is given in [7, Ch. 7]. MATLAB ${ }^{\circledR}$ code for the analytical version of the model is also available on request.

The approximation by an infinite pulse train constitutes the only difference between the stochastic model in Fig. 1 suitable for Monte Carlo simulation and the analytical version of the model. In order to test the accuracy of this analytical approximation, we conducted Monte Carlo simulations of the stochastic model. Responses to 10-, 20-, and 100-ms pulse trains of rates $200 \mathrm{pps}$ and $600 \mathrm{pps}$ were simulated and compared with the results of the analytical approximation. For a 10-ms pulse train, the analytical description approximates the response at 600 pps very well and the response at 200 pps almost exactly (the correlation coefficient $=1.00$ in both cases). Extending the pulse train duration to just $20 \mathrm{~ms}$ (four pulses at $200 \mathrm{pps}$ and 12 pulses at $600 \mathrm{pps}$ ) improves the accuracy considerably, and for a 100-ms pulse train, the analytical expressions provide an almost exact description of the model. This indicates that the accuracy of the analytical descriptions is relatively insensitive to the length of the pulse train, i.e., to the accuracy of the approximation by an infinite pulse train.

Furthermore, our implementation of the analytical approximation takes just $0.04 \%$ of the time required by the Monte Carlo simulation to compute 500 iterations for a 100 -ms pulse train. While the mean of the Monte Carlo stimulation output converges rapidly, giving an accurate estimate after only 100 iterations, the variance has still not converged completely after 500 iterations. The sample mean from the Monte Carlo simulations will converge at a rate of $\sqrt{N}$, where $N$ is the number of iterations, and the sample variance will converge more slowly, at a rate of approximately $\sqrt{(N+1) / 2}[23$, pp. 108]. Therefore, no results of the Monte Carlo simulations are presented here. Instead, predictions of the physiological pulsetrain data by the analytical version of the model are presented in Section III-C.

\section{Prediction of Pulse-Train Response}

Using the analytical version of the model described in Section III-B, we predict both the mean and variance of the discharge rate, for the stochastic pulse train model where fiber threshold and RS are determined from the first-pulse response as described in [1], and for the equivalent deterministic model (i.e., RS is set to zero). Given the values of threshold and $\mathrm{RS}$ from fits to the first-pulse response, it simply remains to choose an appropriate refractory function.

The single-pulse response was calculated by investigating the response of each neuron to the first pulse in a pulse train. It is, therefore, possible to directly calculate the refractory function that produces the best fit to the discharge rate data for the remainder of the pulse train. However, discharge rate data at a range of stimulation rates were collected for

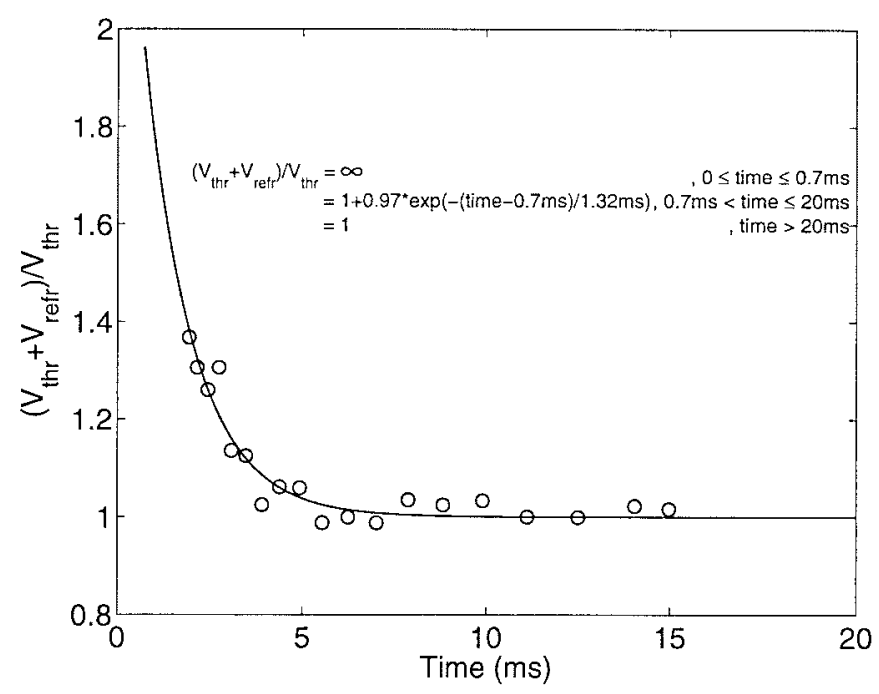

Fig. 2. Relative increase in threshold due to refractory effects: physiological data (o) and exponential least-squares fit (solid line).

only a few neurons in this data set. Consequently, it is infeasible to estimate the range of refractory functions in AN fibers, for use in our total AN model. Therefore, we use a standard refractory function for all neurons, which we derive by fitting an exponential function to the cat data from [13, Fig. 3-5]. These data were collected using monophasic pulses, but produce an exponential function fit similar to that of biphasic pulse data [12]. Our standard refractory function is plotted in Fig. 2 as the relative increase in threshold, i.e., $\left(V_{\text {thr }}+V_{\text {refr }}\right) / V_{\text {thr }}$, along with its analytical description. Note that the absolute refractory period lasts $0.7 \mathrm{~ms}$, after which the relative refractory period begins with a time-constant of $1.32 \mathrm{~ms}$, with the refractory effects lasting for a total period of $20 \mathrm{~ms}$. The effect of refractory function choice is investigated in the next section.

1) Mean Discharge Rate: Plotted in Fig. 3 are mean discharge rates for [9, Neuron 3-21] in response to 100,200 , $300,400,600$, and 800 pps trains of $100-\mu \mathrm{s} /$ phase biphasic pulses. If a deterministic response is assumed, then one may readily attribute all of a fiber's dynamic range to interpulse effects such as refractory effects. We can test this assumption by comparing the mean discharge rate data with the discharge probability for the first pulse in the pulse train, i.e., with a response that is not subject to interpulse effects. Thus, also plotted in Fig. 3 is the first-pulse discharge probability [1] of Neuron 3-21 (dotted lines) scaled to indicate what the pulsetrain response would be if interpulse interactions were not present, i.e., saturation discharge rate $=$ pulse rate. While interpulse effects do indeed increase the dynamic range at higher pulse rates, much of the dynamic range for pulse-train stimuli is due to stochastic activity, particularly for the lower discharge rates.

Note that there were a good deal of responses at the higher stimulus levels for which no data were collected. As the stimulus amplitude was increased, the neurophysiologists would stop collecting data once the discharge rate equaled the stimulus's pulse rate. This was done because they had previously found that the spike rate would remain nearly 


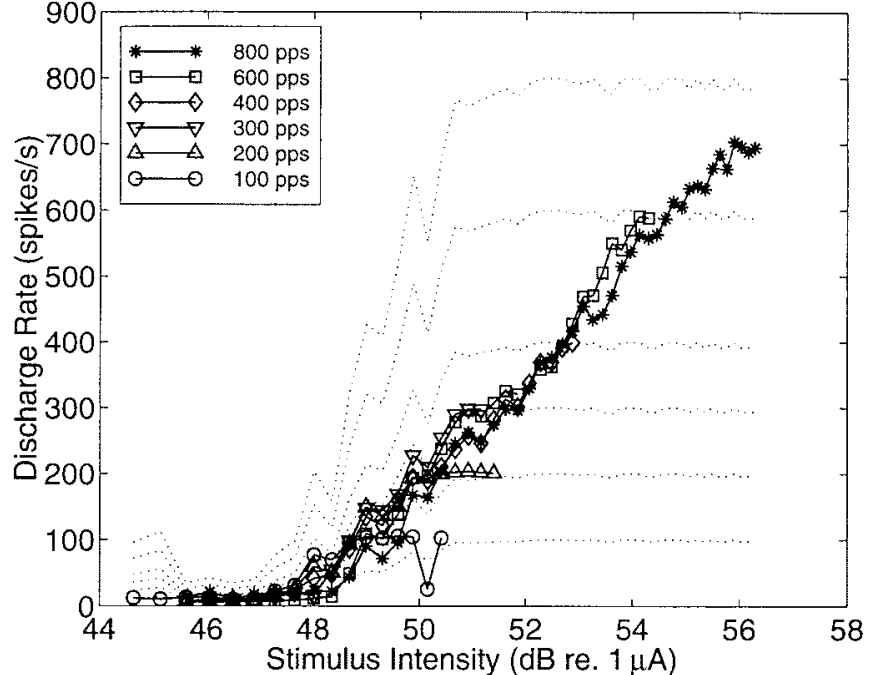

Fig. 3. Mean discharge rates for [9, Neuron 3-21] at pulse rates from 100 to $800 \mathrm{pps}$ with a pulse width (phase duration) of $100 \mu \mathrm{s} / \mathrm{phase}$. Dotted lines are the first-pulse data of Neuron 3-21 scaled to indicate what the pulse-train response would be if interpulse interactionswere not present, i.e., saturation mean discharge rate $=$ pulse rate.

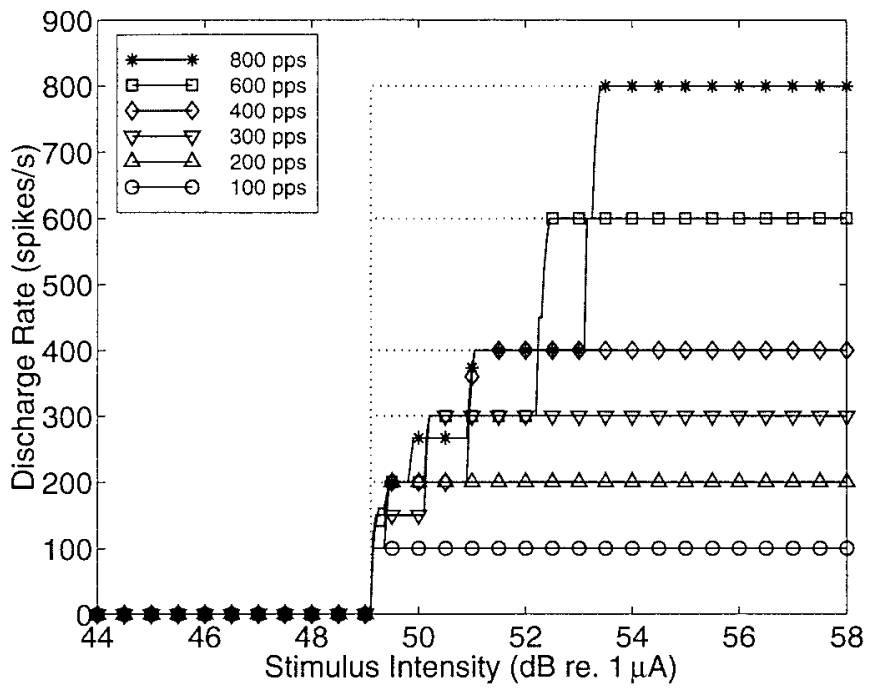

Fig. 4. Mean discharge rates for the deterministic version of the model at pulse rates of $100,200,300,400,600$, and 800 pps with a pulse width of $100 \mu \mathrm{s} / \mathrm{phase}$. Dotted lines indicate mean discharge rates without refractory effects, i.e., single-pulse model predictions.

constant and equal to the pulse rate at these higher stimulus levels.

In Fig. 4, we plot the deterministic pulse-train model's predictions of these data (solid lines). The dotted lines represent the same predictions without refractory effects, obtained from the deterministic single-pulse model presented in [1]. When compared to the data in Fig. 3, the inclusion of the refractory mechanism is seen to improve the model's prediction of the I/O function slope for each pulse rate, but it still does not reach the slope of the physiological data and the shapes of the curves are inaccurate.

Plotted in Fig. 5 are predictions of the mean discharge rates for the stochastic pulse-train model. The dotted lines repre-

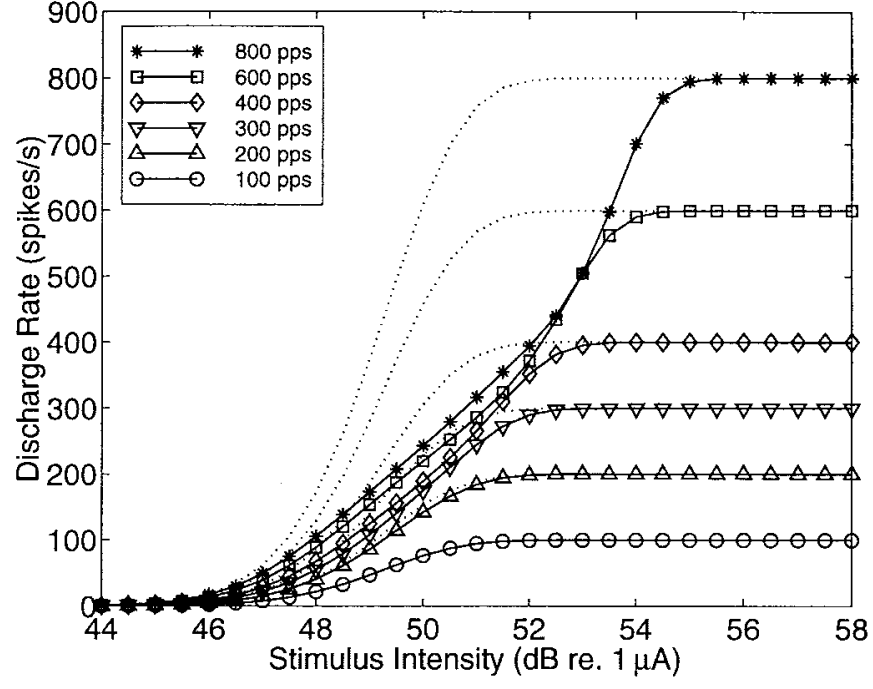

Fig. 5. Mean discharge rates for the stochastic model at pulse rates of 100 , $200,300,400,600$, and 800 pps with a pulse width of $100 \mu \mathrm{s} /$ phase. Dotted lines indicate mean discharge rates without refractory effects, i.e., single-pulse model predictions.

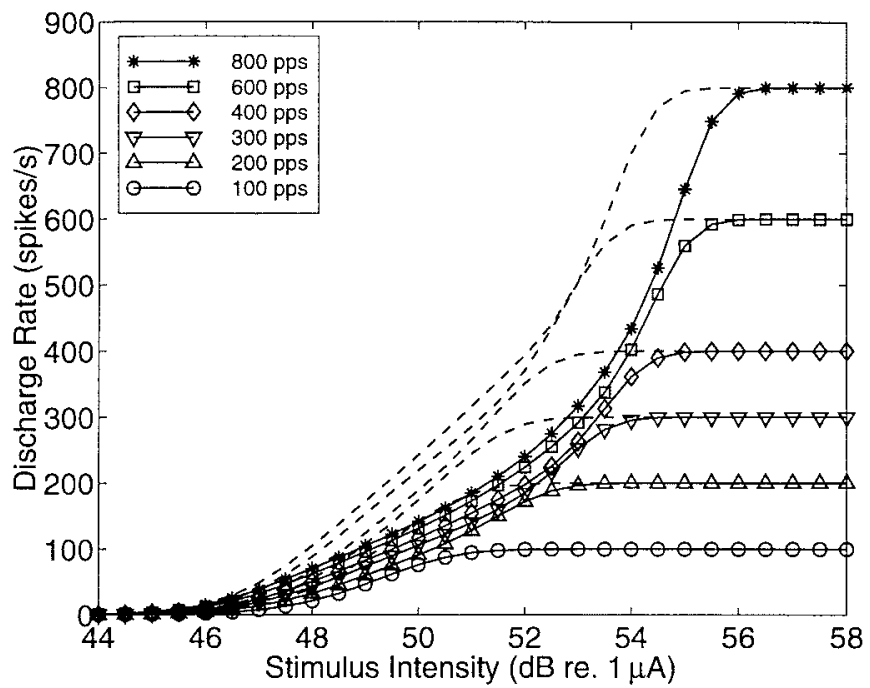

Fig. 6. Mean discharge rates for the stochastic model with a modified refractory function (absolute refractory period $=1 \mathrm{~ms}$; time-constant $=2 \mathrm{~ms}$ ) at pulse rates of $100,200,300,400,600$, and 800 pps with a pulse width of $100 \mu \mathrm{s} /$ phase. Dashed lines indicate mean discharge rates for the standard refractory function (from Fig. 5).

sent the same predictions without refractory effects, obtained from the stochastic single-pulse model presented in [1]. Both the shape of the curves and their slopes better predict the physiological data than the deterministic model does.

We have also investigated the effects of the choice of refractory function. Plotted in Fig. 6 are mean discharge rates where the refractory function has been modified to have an absolute refractory period of $1 \mathrm{~ms}$ and a time-constant of $2 \mathrm{~ms}$. For comparison, we have overlaid the discharge rate functions for the standard refractory function from Fig. 5 (dashed lines). The modified refractory function produces a slightly better prediction of the overlap of the physiological discharge rate versus intensity curves at discharge rates below approximately 


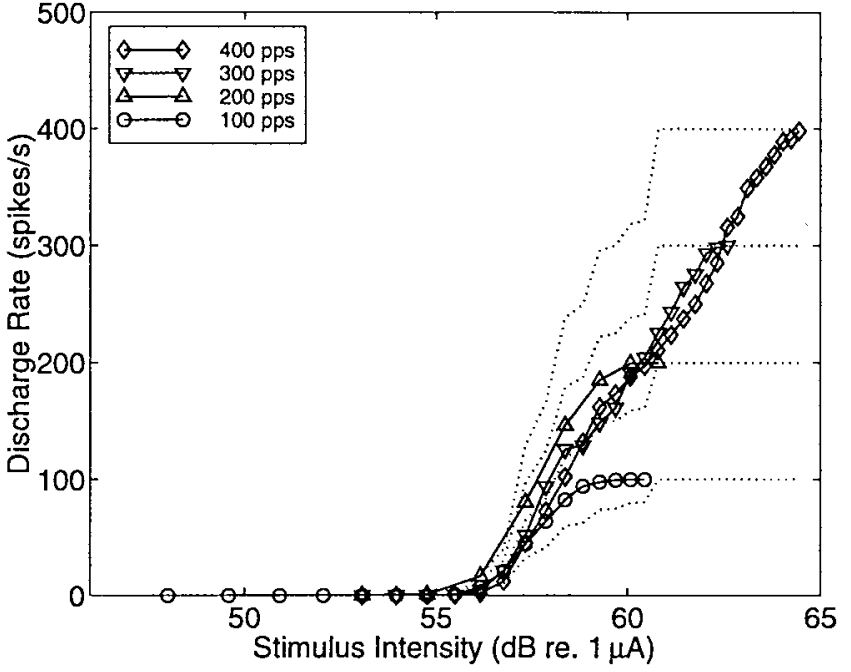

(a)

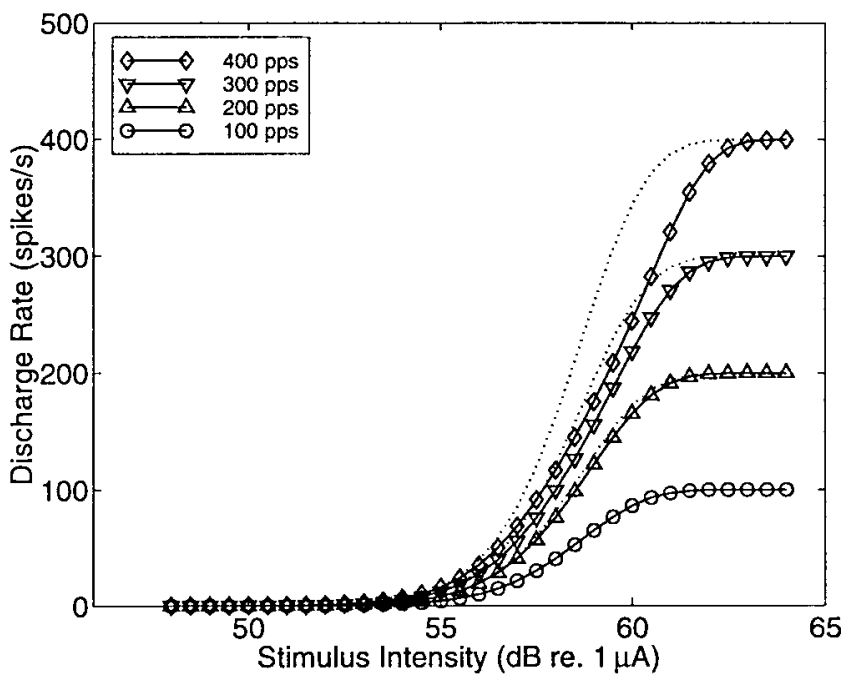

(b)

Fig. 7. Mean discharge rate data and model predictions for [9, Neuron 3-10] at pulse rates from 100 to $400 \mathrm{pps}$ with a pulse width of $200 \mu \mathrm{s} / \mathrm{phase}$. (a) Mean discharge rate data (solid lines) from Neuron 3-10. Dotted lines are the first-pulse data of Neuron 3-10 scaled to indicate what the pulse-train response would be if the interpulse interactions were not present, i.e., saturation mean discharge rate $=$ pulse rate. (b) Stochastic model predictions using the standard refractory function (solid lines). Dotted lines indicate mean discharge rates without refractory effects.

200 spikes/s than the standard refractory function does. However, the shapes of the curves and the slopes appear to be less accurate.

Mean discharge rate data at stimulation rates of 100, 200, 300 and $400 \mathrm{pps}$ from Neurons 3-10 (see Fig. 7) and [9, Neurons 3-29] were also investigated, yielding similar results.

2) Variance of Discharge Rate: In Fig. 8 discharge rate variances (o) are plotted for [9, Neuron 3-21] at pulse rates of 200 and 600 pps. To allow comparison across different pulse rates, both the mean and variance dischargerate data are normalized (divided by the pulse rate). This normalization produces the discharge probability/pulse and the variance per pulse respectively. It can be seen that with increasing pulse rate the variance is compressed around the

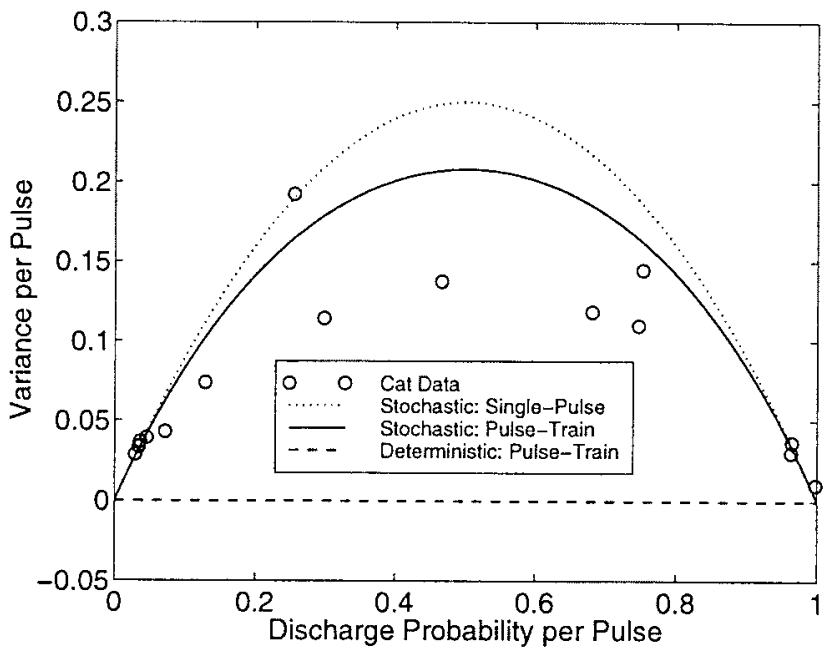

(a)

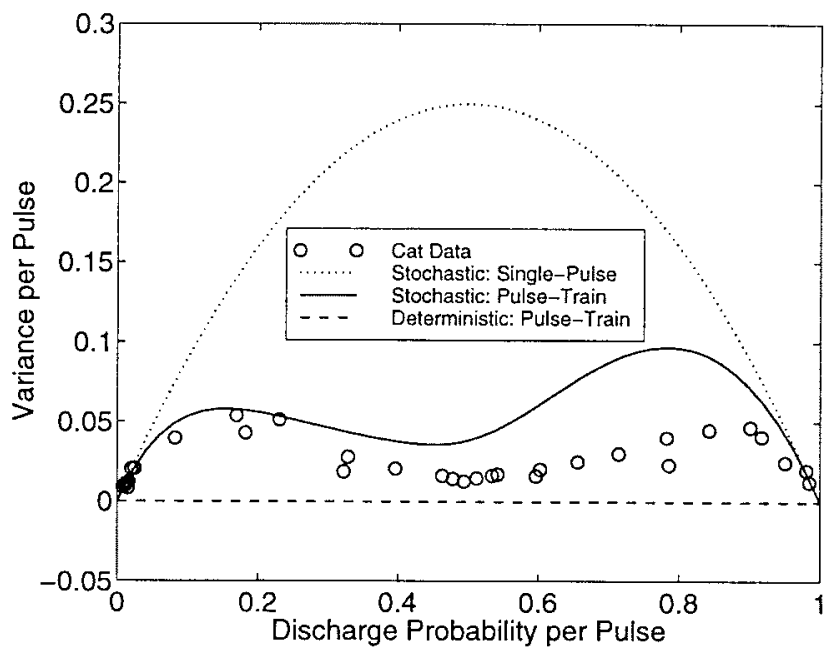

(b)

Fig. 8. Variance per pulse versus discharge probability per pulse (o) for [9, Neuron 3-21] with a pulse width of $100 \mu \mathrm{s} /$ phase at (a) $200 \mathrm{pps}$ and (b) 600 pps. Also plotted are single-pulse stochastic model predictions (dotted lines) of these data, the stochastic model predictions (solid lines) and the deterministic model predictions (dashed lines).

point of 0.5 discharge probability per pulse. The single-pulse version of the stochastic model (dotted line) overestimates the variance, the error increasing with pulse rate. The deterministic model (dashed line), on the other hand, cannot predict the variance at all. The pulse-train stochastic model (solid line) provides the best prediction of the variance data. This was found to be true also for pulse rates of $100,300,400$, and 800 pps.

Just as we investigated the effects of refractory function choice on the mean discharge rate, we also investigate its effect on the prediction of the variance data. In Fig. 9 the discharge rate variance data for $200 \mathrm{pps}$ (o) and 600 pps (x) are replotted from Fig. 8, along with the model predictions with the standard refractory function (dotted lines). Newly plotted are predictions of these data by the stochastic model with a modified refractory function (solid lines)—absolute refractory period $=1 \mathrm{~ms}$; time-constant $=2 \mathrm{~ms}$. It can be seen that the change in refractory 


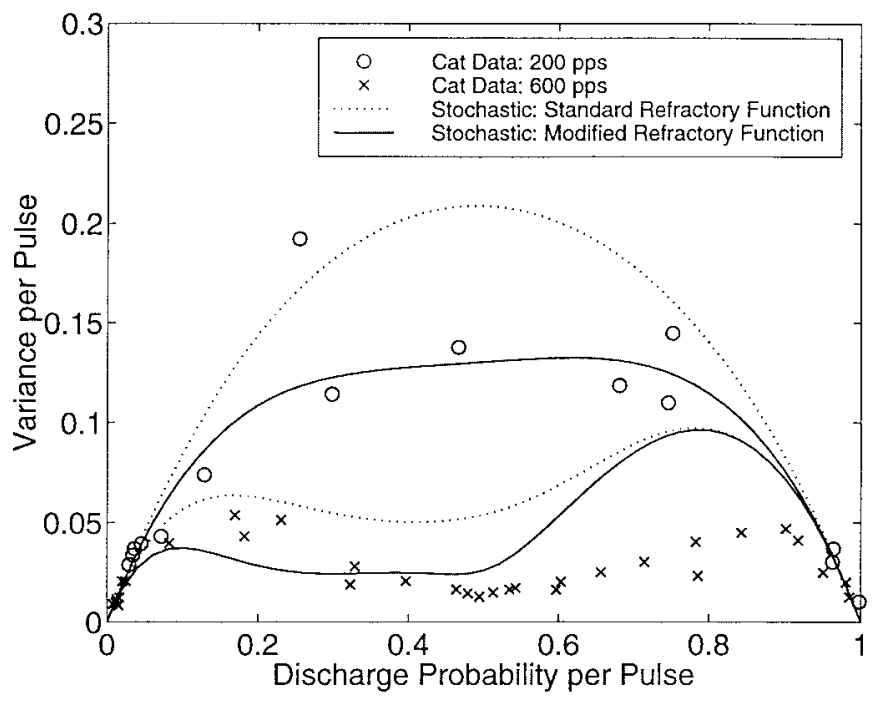

Fig. 9. Sensitivity to refractory function: The variance data for $200 \mathrm{pps}$ (o) and 600 pps (x) are replotted from Fig. 8, along with the model predictions with the standard refractory function (dotted lines). Newly plotted are predictions of these data by the stochastic model with a modified refractory function (solid lines).

function time-constants improves the model predictions except for discharge probabilities above 0.6 in the $600 \mathrm{pps}$ case.

Variance of discharge rate data at stimulation rates of 200 and 400 pps from Neurons 3-10 (see Fig. 10) and [9, Neurons 3-29] were also investigated, yielding similar results.

\section{TOtal Auditory NerVe Response}

The output of our model of total auditory nerve response developed in [1, Section IV] is the discharge probability for each fiber, and the summed response is well approximated by a Poisson distribution if the mean summed response is less than 15 and by a Gaussian if it is greater or equal to 15 . For pulse-train stimuli, the output of the population model simply becomes the mean and variance in discharge rate for each fiber. With regards to the summed response, although for responses to pulse trains the individual fibers are described by renewal processes rather than Bernoulli processes, application of the central limit theorem [24] shows that the summed response will still be well approximated by a Gaussian distribution if the mean is large enough $(\geq 15)$. If the mean is less than 15 , the Poisson distribution approximation of the summed response will worsen if one or more fibers diverge from behaving like a Bernoulli process. As is evident from Figs. 8 and 10, the renewal process model of a fiber's behavior (stochastic pulse-train model) differs from a Bernoulli process (stochastic single-pulse model) only when its discharge probability per pulse becomes larger than about 0.05 . For total cochlear spike counts of less than 15, fiber discharge probabilities above 0.05 could only occur if: i) the density of surviving AN fibers is very low, or ii) the stimulating electrode is very selective and, therefore, effectively stimulates only a small number of fibers.

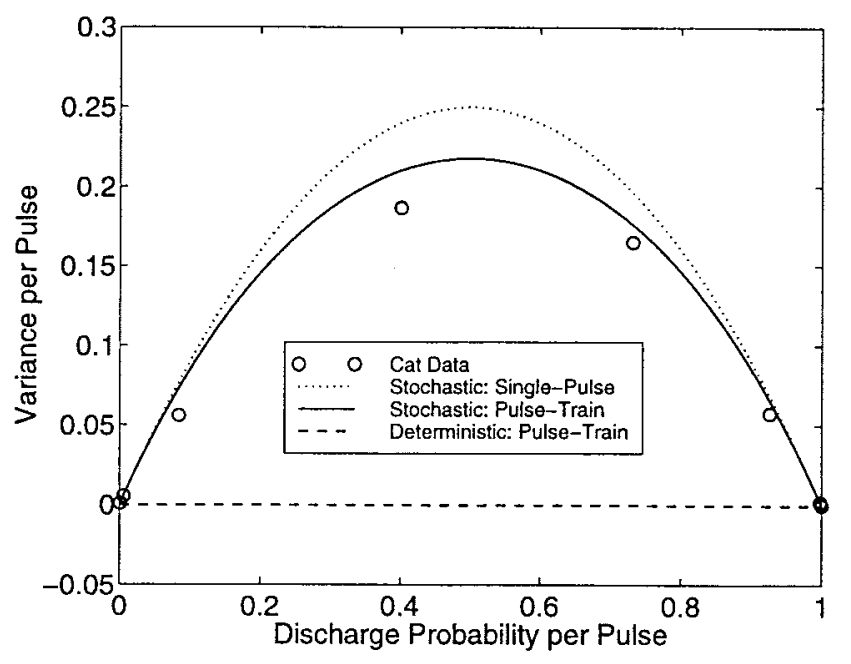

(a)

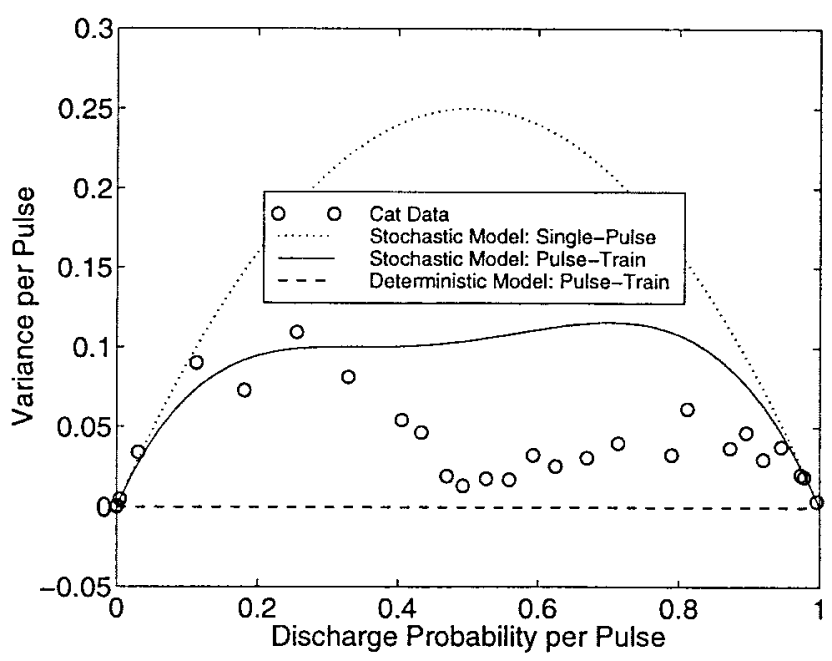

(b)

Fig. 10. Variance per pulse versus discharge probability per pulse (o) for [9, Neuron 3-10] with a pulse width of $100 \mu \mathrm{s} /$ phase at (a) $200 \mathrm{pps}$ and (b) 400 pps. Also plotted are single-pulse stochastic model predictions (dotted lines) of these data, stochastic model predictions (solid lines) and deterministic model predictions (dashed lines), the latter two using the standard refractory function.

\section{DISCUSSION}

We have shown that a deterministic model is a poor description of a fiber's response, in light of the improvements in the prediction of physiological data when a stochastic component of response is added to the neural model. Specifically, we have found that a simple stochastic model can accurately predict the mean and variance of the discharge rate in response to pulse trains. Our results suggest that in the case where a uniform refractory function is assumed across all neurons, just two parameters (threshold and RS) can well describe the response of an auditory nerve fiber to single biphasic pulses and pulse trains. We have shown that an even more precise description may be achieved by estimating a specific refractory function for each neuron. However, the improved accuracy gained by estimating refractory functions for each neuron may not justify the additional parameters required to describe each neuron in the total auditory nerve model. 
The analytical descriptions of single fiber response to pulse trains used in this paper provide a simple and computationally efficient method of modeling the response of a large-scale population of AN fibers to trains of evenly timed, uniform pulses. Furthermore, the output of the model, being a mean and a variance for each neuron with a known distribution, allows the direct use of signal detection theory to determine the resolution with which the AN can convey information about an electrical stimulus.

As was the case for the single-fiber model of [1], the pulse-train model presented in this paper may also be suitable for functional electrical stimulation of other types of nerve fibers. However, use of this model for a paradigm other than stimulation of the AN by a cochlear implant would require validation of the assumptions and approximations of this paper for the particular stimulus and neural properties of that paradigm.

The analytical approximation was developed to describe responses to trains of evenly timed, uniform pulses, as are commonly used in psychophysical and physiological investigations. However, current speech processing strategies used in cochlear implants may produce trains of pulses that vary in amplitude over the duration of the train. Neither the analytical descriptions, nor the methods used in deriving them, can be directly applied to simulate the response to such pulse trains. Monte Carlo simulations of the model illustrated in Fig. 1 could be used instead to estimate the neural response.

Further physiological and modeling studies would be helpful in refining and extending a number of aspects of our model-specific suggestions follow.

Discharge rate data at a range of stimulation rates were only available for three neurons in the cat data analyzed in this paper. It would be desirable to compare the model to more physiological data, for further verification of the model. Additional data could also help in further refining the model. In particular, an estimate of the distribution of refractory functions in the cat $\mathrm{AN}$ could be obtained if more data were available.

The stimulation rates investigated in this paper were all 800 pps or lower. Development of speech processing strategies utilizing significantly higher stimulation rates is currently being investigated by a number of cochlear implant research groups. It would, therefore, be useful to extend our model to describe physiological data collected at these higher stimulation rates when such data become available.

Furthermore, much of the neurophysiology and, as a consequence, much of the modeling in this paper emphasizes moderate to high discharge probabilities per pulse. Implant users may be operating at considerably lower discharge probabilities, particularly when receiving continuous stimulation from a multichannel speech processor [6]-[8]. It may, therefore, be useful to collect further data at stimulus intensities that produce low discharge probabilities per pulse.

Further investigation into the behavior of membrane noise following both subthreshold and suprathreshold stimulation is required. Single-fiber data from the cat $\mathrm{AN}$ and one stochastic model [13, Fig. 5-7] indicate that the membrane noise does not change significantly during the refractory period. However, membrane noise was seen to increase following a subthreshold pulse, i.e., in response to a depolarization that does not generate an action potential. This may explain why, for mid to high pulse rates, the variance per pulse in our cat data is slightly greater for discharge probabilities per pulse below 0.5 than for discharge probabilities above 0.5 [e.g., see Fig. 10(b)]: for discharge probabilities per pulse below 0.5 , the majority of the pulses in a pulse train are effectively subthreshold, producing greater membrane noise and, consequently, greater variance in the response. This phenomenon could be significant if cochlear implants are operating at intensities that produce low discharge probabilities, such that many pulses in a pulse train will effectively be subthreshold.

A different stochastic model [14] has shown that in some cases membrane noise may increase significantly during a portion of the refractory period. Single-fiber data from the cat AN [15] also revealed that some neurons exhibit double-spiking and that the stochastic component of the second spike is significantly increased. This may be a result of the membrane noise increasing by such an amount during the refractory period following the first spike that a second spike is spontaneously generated [15]. These phenomena could also be significant for cochlear implant speech processing strategies and, therefore, further investigation of these phenomena is desirable.

As well as choice of refractory function and the effects of subthreshold and suprathreshold stimulation on noise levels, the other interpulse interactions discussed in Section III-A such as the excitatory effect of subthreshold stimulation on thresholds, but not included in this model, may account for some of the differences seen between the variance data at mid to high discharge rates and our model's predictions of these data (e.g., see Figs. 8 and 10). These other interpulse interactions could be included in our model when they are better understood, and may be modeled directly using our methodology if they do not violate the assumptions and approximations set forth. Additionally, as was the case in [1], a separate stochastic description of action potential latency would be needed to investigate the effects of jitter in theories of coding that are dependent on the exact timing of discharges. Our model is also unable to deal with specific effects occurring in response to pulse trains that are due to neural conduction properties, e.g., anodal or cathodal blocking [25], or the occurrence of abortive spikes as observed by Paintal [26] and simulated with a deterministic cable model by Frijns et al. [27].

In [7] and [8], we used the stochastic single-pulse model of [1] to predict a range of measures of loudness perception, including threshold, uncomfortable loudness, and intensity difference limen. The results of that study suggest that cochlear implants are indeed operating in the region where stochastic activity is significant. In all the cases investigated, the stochastic model predicted psychophysical performance significantly better than the deterministic model did. This suggests that the stochastic single-pulse model is not just more accurate in its prediction of physiological response, but also in its prediction of the resulting behavioral performance. With the pulse-train model presented in this paper, we may now have an appropriate tool for extending this investigation to the prediction of psychophysical data for higher stimulation rates. 


\section{ACKNOWLEDGMENT}

The authors would like to thank J. Tong and R. Shepherd for their part in collecting the physiological data of [9], R. Shepherd, A. Burkitt, L. Cohen and the four anonymous reviewers for invaluable comments on the manuscript, and C. van den Honert and C. Finley for their assistance given to $\mathrm{M}$. White in initial investigations of stochastic activity in the auditory nerve.

\section{REFERENCES}

[1] I. C. Bruce, M. W. White, L. S. Irlicht, S. J. O'Leary, S. Dynes, E. Javel, and G. M. Clark, "A stochastic model of the electrically stimulated auditory nerve: Single-pulse response," this issue, pp. XXX-XXX.

[2] A. A. Verveen and H. E. Derksen, "Fluctuation phenomena in nerve membrane," in Proc. IEEE, vol. 56, pp. 906-916, June 1968.

[3] M. W. White, Design considerations of a prosthesis for the profoundly deaf, Ph.D. dissertetion, Univ. California, Berkeley, CA, 1978.

[4] M. W. White, C. C. Finley, and B. S. Wilson, "Electrical stimulation model of the auditory nerve: Stochastic response characteristics," in Proc. 9th Annu. Conf. IEEE Engineering in Medicine and Biology Society, Boston, MA., 1987, pp. 1906-1907.

[5] I. C. Bruce, L. S. Irlicht, M. W. White, S. J. O'Leary, and G. M. Clark, "Electrical stimulation of the auditory nerve: Prediction of psychophysical performance by a model including stochastic aspects of neural response," in ARO Midwinter Meeting Abstracts, 1997.

[6] I. C. Bruce, L. S. Irlicht, M. W. White, S. J. O'Leary, S. Dynes, E. Javel, and G. M. Clark, "An improved model of electrical stimulation of the auditory nerve," in Cochlear Implants. XVI World Congress of Otorhinolaryngology Head and Neck Surgery, G. M. Clark, Ed. Bologna, Italy: Monduzzi Editore, 1997, pp. 125-130.

[7] I. C. Bruce, Spatiotemporal coding of sound in the auditory nerve for cochlear implants, Ph.D. dissertation, The University of Melbourne, Melbourne, Australia, 1997.

[8] I. C. Bruce, M. W. White, L. S. Irlicht, S. J. O'Leary, and G. M. Clark, "The effects of stochastic neural activity in a model predicting intensity perception with cochlear implants: Low-rate stimulation," submitted for publication.

[9] E. Javel, Y. C. Tong, R. K. Shepherd, and G. M. Clark, "Responses of cat auditory nerve fibers to biphasic electrical current pulses," Ann. Otol. Rhinol. Laryngol., vol. 96, no. Suppl. 128, pp. 26-30, 1987.

[10] S. J. O'Leary, L. S. Irlicht, I. C. Bruce, M. W. White, and G. M. Clark, "Variance of spike rate from auditory nerve following electrical pulsetrain stimulation of cochlea: An experimental and modeling study," submitted to J. Acoust. Soc. Amer., 1998.

[11] P. H. Stypulkowski and C. van den Honert, "Physiological properties of the electrically stimulated auditory nerve. I. Compound action potential recordings," Hear. Res., vol. 14, pp. 205-223, 1984.

[12] C. W. Parkins, "Temporal response patterns of auditory nerve fibers to electrical stimulation in deafened squirrel monkeys," Hear. Res., vol. 41, pp. 137-168, 1989.

[13] S. Dynes, Discharge characteristics of auditory nerve fibers for pulsatile electrical stimuli, Ph.D. dissertation, Massachusetts Inst. Technol., Cambridge, MA, 1996.

[14] J. T. Rubinstein, A. J. Matsuoka, P. J. Abbas, and C. A. Miller, "The neurophysiological effects of simulated auditory prosthesis stimulation," Dept. Otolaryngology - Head and Neck Surgery and Department of Speech Pathology and Audiology, University of Iowa, Iowa City, IA, 2nd Quar. Progress Rep.-NO1-DC-6-2111, 1997.

[15] C. A. Miller, P. J. Abbas, J. T. Rubinstein, and A. J. Matsuoka, "The neurophysiological effects of simulated auditory prosthesis stimulation," Dept. Otolaryngology - Head and Neck Surgery and Department of Speech Pathology and Audiology, University of Iowa, Iowa City, IA, 3rd Quart. Progress Rep.-NO1-DC-6-2111, 1997.
[16] R. Butikofer and P. D. Lawrence, "Electrocutaneous nerve stimulation. II: Stimulus waveform selection," IEEE Trans. Biomed. Eng., vol. BME26, pp. 69-75, Jan. 1979.

[17] J. H. M. Frijns, Cochlear implants-A modeling approach, Ph.D. thesis, Leiden University, Leiden, the Netherlands, 1995.

[18] H. Lecar and R. Nossal, "Theory of threshold fluctuations in nerves," Biophysical J., vol. 11, pp. 1048-1067, 1971.

[19] I. J. Hochmair-Desoyer, E. S. Hochmair, H. Motz, and F. Rattay, "A model for the electrostimulation of the nervus acusticus," Neurosci., vol. 13 , no. 2, pp. 553-562, 1984.

[20] J. T. Rubinstein, "Threshold fluctuations in an N sodium channel model of the node of Ranvier," Biophysical J., vol. 68, pp. 779-785, 1995.

[21] D. R. Cox, Renewal Theory, ISBN 0-412-20570-X, Science Paperbacks. New York: Chapman and Hall, 1962.

[22] W. Feller, An Introduction to Probability Theory and Its Applications, vol. 1, 3rd ed. New York: Wiley Series in Probability and Mathematical Statistics, 1968.

[23] E. S. Keeping, Introduction to Statistical Inference. New York: Van Nostrand 1962

[24] J. L. Devore, Probability and Statistics for Engineering and the Sciences, 2nd ed. Monterey, CA: Brooks/Cole, 1987.

[25] J. B. Ranck Jr., "Which elements are excited in electrical stimulation of mammalian central nervous system: A review," Brain Res., vol. 98, pp. $417-440,1975$

[26] A. S. Paintal, "Conduction properties of normal peripheral mammalian axons," in Physiology and Pathobiology of Axons, S. G. Waxman, Ed. New York: Raven, 1978, pp. 131-144.

[27] J. H. M. Frijns, J. Mooij, and J. H. ten Kate, "A quantitative approach to modeling mammalian myelinated nerve fibers for electrical prosthesis design," IEEE Trans. Biomed. Eng., vol. 41, pp. 556-566, 1994.

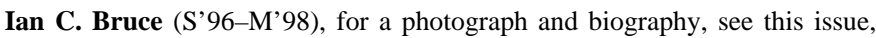
p. 628.

Laurence S. Irlicht (S'96-M'98), for a photograph and biography, see this issue, p. 628 .

Mark W. White (M'79), for a photograph and biography, see this issue, p. 628.

Stephen J. O'Leary, for a photograph and biography, see this issue, p. 628.

Scott Dynes, for a photograph and biography, see this issue, p. 628.

Eric Javel, for a photograph and biography, see this issue, p. 628 .

Graeme M. Clark, for a photograph and biography, see this issue, p. 629. 


\section{University Library}

\section{- M M N E R VA A gateway to Melbourne's research publications}

Minerva Access is the Institutional Repository of The University of Melbourne

Author/s:

Bruce, Ian C.;Irlicht, Laurence S.;White, Mark W.;O'Leary, Stephen J.;Dynes, Scott;Javel, Eric;Clark, Graeme M.

Title:

A stochastic model of the electrically stimulated auditory nerve: pulse-train response

Date:

1999

\section{Citation:}

Bruce, I. C., Irlicht, L. S., White, M. W., O'Leary, S. J., Dynes, S., Javel, E., et al. (1999). A stochastic model of the electrically stimulated auditory nerve: pulse-train response. IEEE Transactions on Biomedical Engineering, 46(6), 630-637.

Persistent Link:

http://hdl.handle.net/11343/27531 